\title{
Mise en évidence d'un composé à hétérocycle azoté responsable d'un défaut d'arôme dans le Gruyère de Comté
}

\author{
par \\ J.P. DUMONT, Sylviane ROGER et J. ADDA
}

Lorsque l'on considère les différents défauts de flaveur susceptibles d'affecter la qualité du Gruyère de Comté, il apparaît possible de les classer selon deux types principaux :

- D'une part les défauts qui se manifestent de façon plus ou moins fréquente (goût piquant, par exemple) qui peuvent être qualifiés de classiques.

- D'autre part, les défauts qui apparaissent occasionnellement de façon très localisée et qui conduisent à des produits présentant des goûts véritablement anormaux.

C'est dans cette deuxième catégorie qu'il faut ranger le défaut d'arôme qui s'est développé au cours de l'affinage d'un Gruyère de Comté provenant d'une petite fromagerie du Jura. Ce fromage, dont l'aspect à la coupe était normal possédait une odeur décrite par les experts comme odeur de pomme de terre crue ou odeur de cave. Le défaut, apparu à un stade précoce de l'affinage, était perceptible principalement au niveau de la croûte et de la région subcorticale.

\section{MATERIEL ET METHODES}

\section{a) Echantillonnage et extraction}

$2,5 \mathrm{~kg}$ environ de fromage ont été prélevés sur la partie externe de la meule, sur une épaisseur de 2 à $3 \mathrm{~cm}$.

L'échantillon réparti en lots de $125 \mathrm{~g}$ a été conservé à $-30^{\circ} \mathrm{C}$ jusqu'au moment de l'analyse. Chacune de ces portions a été râpée à l'état congelé puis soumise à une distillation sous vide poussée suivant une technique précédemment décrite [14]. 
Le distillat aqueux obtenu est saturé avec du chlorure de sodium, puis amené à $\mathrm{pH} 1,0$ par addition de $\mathrm{HCl} 1 \mathrm{~N}$. Il est alors extrait par un petit volume d'éther purifié. On obtient ainsi les composés neutres et acides. Le distillat aqueux est ensuite amené à $\mathrm{pH} 11,0$ par addition de $\mathrm{NaOH} \mathrm{N}$ et extrait de nouveau par l'éther éthylique ce qui permet d'obtenir les composés possédant un caractère alcalin.

Les extraits éthérés sont séchés sur sulfate de sodium anhydre puis concentrés sous colonne de Vigreux.

\section{b) Analyses chromatographiques}

Les analyses ont été effectuées sur un appareil Girdel 75 CD PT modifié de façon à obtenir le tracé de la détection par ionisation de flamme tout en évaluant simultanément l'intensité et la qualité olfactive des différents composés séparés en sortie de colonne (cette modification permet également le piégeage de différentes fractions).

Suivant les cas, l'appareil était équipé soit d'un détecteur à ionisation de flamme, soit d'un détecteur thermoionique. Nous avons utilisé une colonne en acier inoxydable de $3 \mathrm{~m}$ de long et de $1 / 8$ de pouce de diamètre externe remplie de chromosorb G (60-80 mesh) AW-DMCS, imprégné de 5 p. 100 de Carbowax 20 M-TPA (Applied Sciences) purifié par nos soins.

La température d'analyse était augmentée de façon linéaire à raison de $2^{\circ} \mathrm{C}$ par minute, de $50^{\circ} \mathrm{C}$ à $165^{\circ} \mathrm{C}$ après une période isotherme de $10 \mathrm{mn}$ à $50^{\circ} \mathrm{C}$.

D'autres analyses ont également été réalisées sur une colonne de $3 \mathrm{~m}$ de long remplie de chromosorb W-80/100 imprégné de $10 \mathrm{p} .100$ de silicone $\mathrm{SF} 96$, chauffée de $30^{\circ} \mathrm{C}$ à $180^{\circ} \mathrm{C}$ à raison de $1,5^{\circ} \mathrm{C}$ par minute.

\section{c) Spectromètre de masse}

Les extraits sont analysés sur un appareil Girdel 3000 couplé à un spectromètre de masse à faible résolution (AEI MS 20 organique).

Les conditions chromatographiques utilisées étaient identiques à celles de l'analyse olfactométrique et les spectres, enregistrés à $70 \mathrm{eV}$, ont été identifiés en ayant recours aux données publiées dans la littérature.

\section{RESULTATS}

Il est apparu que l'odeur caractéristique présentée par le distillat aqueux disparaissait lorsqu'il était amené à un $\mathrm{pH}$ acide. Ceci donnait à penser que le ou les composés responsables du défaut possédait un caractère alcalin. 
En conséquence nous avons isolé les composés basiques après extraction préalable des substances neutres et acides. L'extrait ainsi obtenu présentait une odeur intense reproduisant le défaut étudié. L'analyse chromatographique a révélé que cet extrait contenait un nombre important de composés. L'utilisation d'un détecteur thermoionique, puis le couplage chromatographie en phase gazeuse-spectrométrie de masse ont montré qu'il s'agissait de substances azotées (tab 1 ; fig. 1) et non pas de contaminants résultant d'une extraction insuffisante des composés neutres.

\section{$T A B L E A U 1$}

Composés mis en évidence dans la fraction alcaline du Comté défectueux

\begin{tabular}{|c|c|c|}
\hline Peak $n^{\circ}$ & & Spectre de référence \\
\hline 1 & Pyridine & A \\
\hline 2 & 2,5-diméthyl pyrazine & B \\
\hline 3 & Diméthyl pyridine & A \\
\hline 4 & Triméthyl pyridine & A \\
\hline 5 & Triméthyl pyrazine & $\mathrm{C}$ \\
\hline 6 & 2-méthyl-5-éthyl pyridine & A \\
\hline 7 & 2-éthyl-5,6-diméthyl pyrazine & $\mathrm{D}$ \\
\hline 8 & Tétraméthyl pyrazine & $\mathrm{E}$ \\
\hline 9 & $\begin{array}{l}\text { Inconnu } \\
\mathrm{m} / \mathrm{e} 112-71-43\end{array}$ & - \\
\hline 10 & 2-éthyl-3,5,6-triméthyl pyrazine & $\mathrm{D}$ \\
\hline 11 & & - \\
\hline 12 & $\begin{array}{l}\text { Inconnu } \\
\text { m/e 84-43-127-57-56-70-58-44 }\end{array}$ & - \\
\hline 13 & Diméthyl diéthyl pyrazine & - \\
\hline 14 & Indole & A \\
\hline
\end{tabular}
A = CORNu et Massot (1966).
$\mathrm{B}=$ FERRETTI et al. (1970).
C $=$ MaSON et al. (1966).
D = Van PraAg et al. (1968).
$\mathrm{E}=$ Ferretti et Flanagan (1971).

Une analyse identique effectuée sur un Gruyère de Comté ne présentant pas le défaut étudié et pris comme témoin a montré que, pour la plupart, ces substances azotées n'y semblent pas habituellement présentes (fig. 2). Cependant, comme aucun des composés mis 


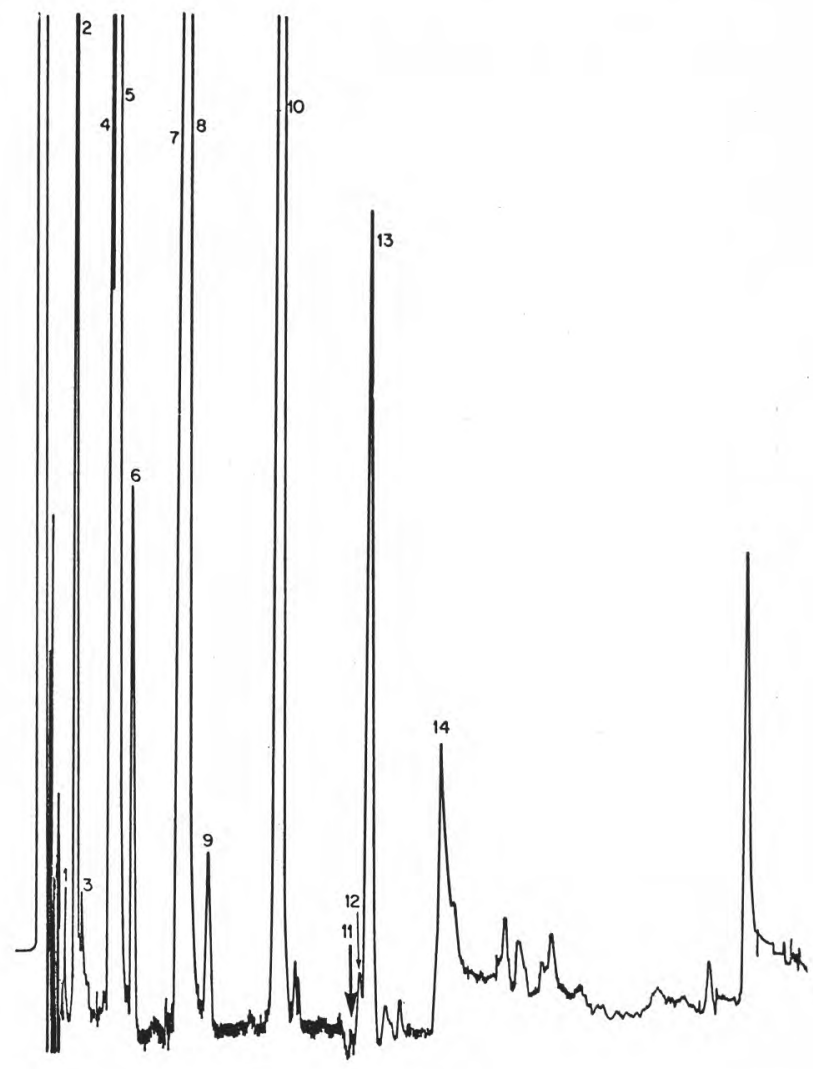

fig. 1

Composés alcalins présents dans le Comté défectueux.

Conditions opératoires :

- Séparation sur colonne de SF 96.

- Détection par détecteur thermoionique.

en évidence n'était connu pour posséder une odeur rappelant celle du fromage défectueux on en a déduit que la substance responsable devait être présente en quantités ne permettant pas sa détection chromatographique dans les conditions utilisées. Une séparation chromatographique avec détection olfactive des composants élués a été réalisée sur deux colonnes de polarité différente et a permis de déterminer avec précision le volume de rétention d'un composé ayant une odeur typique et intense ( ic $\mathrm{n}^{\circ} 11$ de la figure 1 ). 


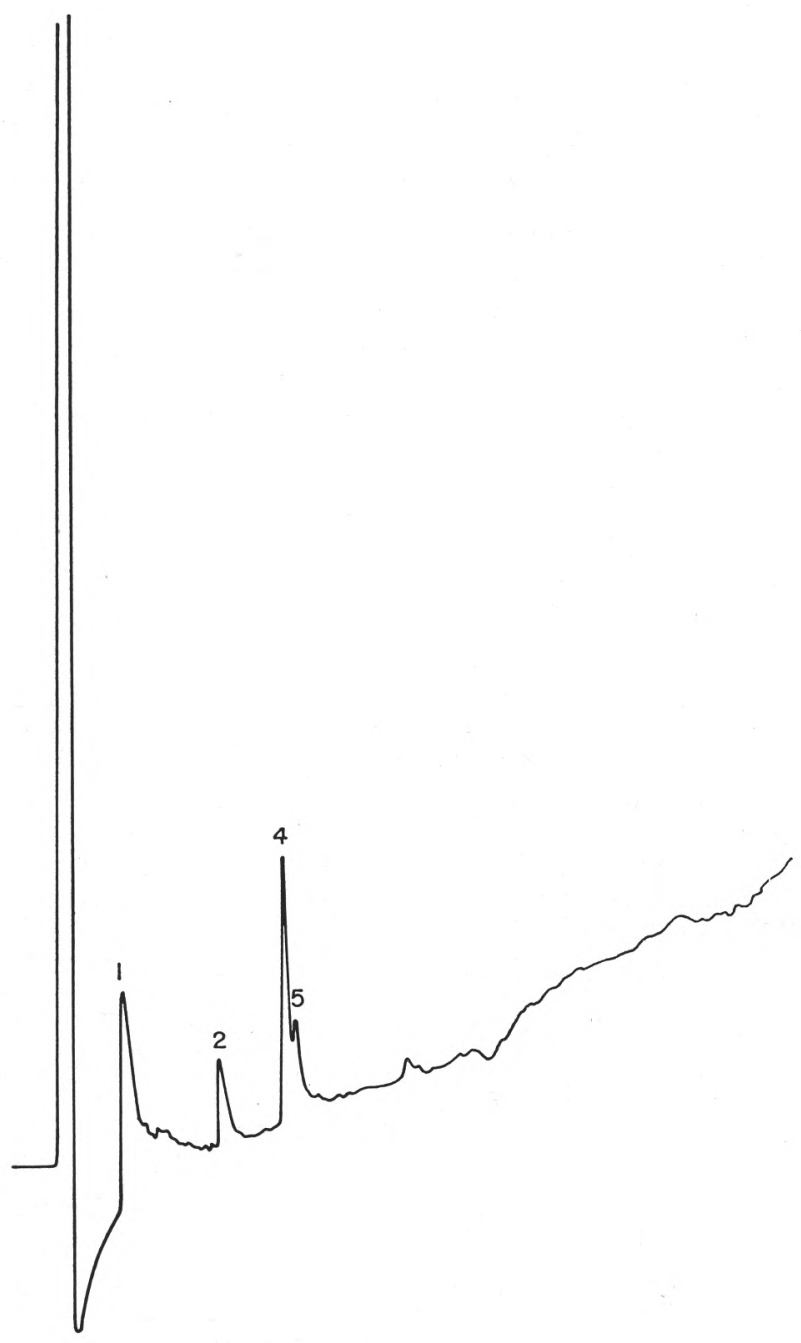

fig. 2

Composés alcalins présents dans un Comté de bonne qualité

Conditions opératoires identiques à celles utilisées pour l'analyse du Comté défectueux (fig. 1).

La numérotation des pics est celle qui a été utilisée dans le tableau 1. 
L'étude en spectrométrie de masse a permis de mettre en évidence un pic majeur à $\mathrm{m} / \mathrm{e}=123$ mais il est apparu qu'une substance, différente dans les deux cas, était éluée en même temps que le corps recherché.

Une chromatographie semi-préparative sur colonne de Carbowax $20 \mathrm{M}$ a permis de piéger de façon additive le composé responsable du défaut. Après rinçage du piège par de l'éther éthylique et concentration, la fraction ainsi récupérée a été réinjectée sur la colonne SF 96. On a pu ainsi obtenir un spectre assez pur qui est présenté dans le tableau 3.

\section{$T A B L E A U 2$}

Spectre de masse de la 2-isobutyl-3-méthoxy pyridine

(Pittet et Hruza (1974), J. Agr. Food Chem., 22, 264-269)

\begin{tabular}{c|c|c|c|c|c|c}
\hline $\mathrm{m} / \mathrm{e}$ & 123 & 122 & 39 & 93 & 150 & 165 \\
\cline { 2 - 4 } & 100 & 43 & 37 & 33 & 24 & 6 \\
\cline { 2 - 4 } p. 100 & 100 & & & & \\
\hline
\end{tabular}

TABLEAU 3

Spectre de masse du composé inconnu (pic 11 de la figure 1)

\begin{tabular}{|c|c|c|c|c|c|c|}
\hline $\mathrm{m} / \mathrm{e}$ & 123 & 122 & 39 & 136 & 65 & 93 \\
\hline p. 100 & 100 & 50 & 50 & 38 & 29 & 27 \\
\hline
\end{tabular}

\begin{tabular}{c|c|c|c|c|}
\hline $\mathrm{m} / \mathrm{e}$ & 108 & 92 & 150 & 151 \\
\hline & & 26 & 14 & 11 \\
\cline { 2 - 3 } & 27 & 26 & & \\
\hline
\end{tabular}

Ce spectre est très voisin de celui rapporté par Pittet [10] pour la 3-méthoxy-2-isobutyl pyridine (tab. 2) composé de masse molécu- 
laire M 165. La masse moléculaire du composé mis en évidence étant de 151 et les fragments de m/e 122 et 123 pouvant être respectivement attribués aux ions :

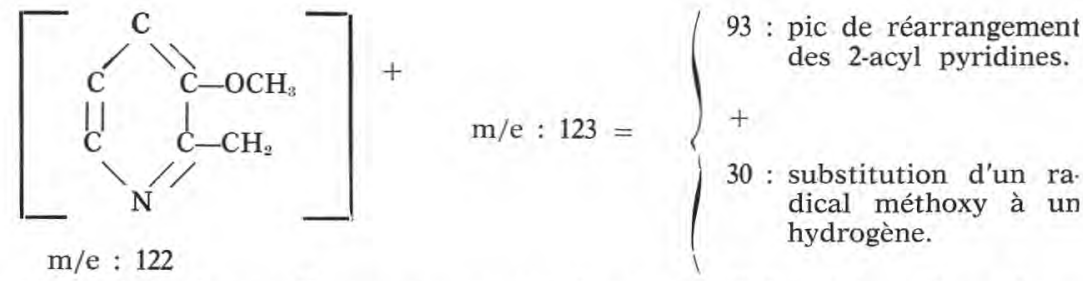

ceci conduit à attribuer au corps inconnu l'identité présumée : 3-méthoxy-2-propyl pyridine.

\section{DISCUSSION}

Les propriétés organoleptiques (odeur caractéristique) de la 3-méthoxy-propyl pyridine n'ont pas été décrites à ce jour. Seule la synthèse de ce composé pourrait permettre de lui attribuer de façon certaine la responsabilité du défaut étudié. Cependant un certain nombre de données viennent étayer cette hypothèse.

Tout d'abord si l'on se rapporte à la théorie de Pittet sur les similitudes qui existent entre les odeurs des composés présentant une même structure chimique, on s'aperçoit que la 3-méthoxy-2-propyl pyridine peut être associée avec la 2-méthoxy-3-propyl pyrazine (et même avec la 2-méthoxy-3-isopropyl pyrazine dont l'odeur de pomme de terre crue est bien connue [11]). Ces composés ont des odeurs qui correspondent assez bien au défaut observé et ont des seuils de perception extrêmement bas (de l'ordre de $10^{-12}$ ).

D'autre part on sait que la 2-méthoxy-3-isopropyl pyrazine a été isolée de produits végétaux [1,9] et qu'elle peut être un métabolite de certains micro-organismes tels Pseudomonas taetrolens [8] et Pseudomonas perolens [7]. On peut donc penser, compte tenu de la similitude de structure entre ce composé et celui que nous pensons avoir identifié, a une origine assez voisine pour ce dernier.

Des analyses microbiologiques effectuées sur le fromage étudié n'ont pas permis d'isoler de souche susceptible d'être rendue responsable du défaut mais il se peut que les micro-organismes incriminés n'aient plus été viables au moment du prélèvement bactériologique.

Il reste cependant un fait que nous ne pouvons expliquer et qui a trait au nombre et à la quantité inhabituels de pyrazines substituées mises en évidence dans l'échantillon. Compte tenu des traitements thermiques très modérés que subit le lait au cours de la fabrication du Gruyère de Comté, il apparaît pratiquement impossible d'attribuer 
la formation de ces pyrazines au mode thermique habituel [12]. Dans ces conditions il faut plutôt attribuer à ces pyrazines une origine microbiologique du même type que celle proposée par Kosuge pour la tétraméthylpyrazine [5].

Tout ceci laisse à penser que la formation de pyrazines par la voie biologique pourrait être un phénomène beaucoup plus courant qu'on ne l'avait cru primitivement.

Reçu pour publication le. 14 mai 1975.

\section{R és u m é}

L'odeur de pomme de terre crue présentée par un fromage de Comté a été attribuée à la fraction alcaline des composés volatils du fromage. Dans cette fraction qui était composée essentiellement de dérivés acylsubstitués de la pyridine et de la pyrazine, il a été possible de mettre en évidence un corps possédant une forte odeur caractéristique du défaut et auquel nous avons attribué l'identité présumée : 3-méthoxy-2-propyl pyridine.

Par sa conformation, ce composé s'apparente à la 2-méthoxy-3isopropyl pyrazine dont l'odeur de pomme de terre crue est bien connue et dont la formation par certains Pseudomonas a été établie récemment.

\section{S u $\mathrm{m} \mathbf{m}$ a r y}

IDENTIFICATION OF A NITROGENOUS HETEROCYCLIC COMPOUND RESPONSIBLE FOR A POTATO-LIKE OFF-FLAVOUR IN GRUYÈRE DE COMTÉ

Several alkylpyrazines and pyridines were got from a Gruyère de Comté exhibiting a strong raw potato-like off-flavour. A compound was found to possess an odor close to the cheese one and was tentatively identificated as 3-methoxy-2-propyl pyridine. Its formation is related to that of 2-methoxy alkylpyrazines by biochemical pathways.

\section{Références bibliographiques}

[1] Buttery (R. G.) and Ling (L. C.) (1973). - Earthy aroma of potatoes. J. Agr. Food Chem., 21, 745-746.

[2] CoRnu (A.) et Massot (R.) (1966). - Index de spectres de masse. P.U.F.

[3] Ferretti (A.) and Flanagan (V. P.) (1971). - The lactose casein browning system : Volatile components. J. Agr. Food. Chem., 19, 245-249.

[4] Ferretti (A.), Flanagan (V. P.) and Ruth (J. M.) (1970). - Non-enzymatic browning in a lactose-casein model system. J. Agr. Food Chem., 18, 13-18. 
[5] Kosuge (T.) and Kamiya (H.) (1962). - Discovery of a pyrazine in a natural product : Tetramethylpyrazine from cultures of a strain of Bacillus subtilis. Nature, 193, 776.

[6] Mason (M. E.), Johnson (B.) and Hamming (M.) (1966). - Flavor components of roasted peanuts. Some low molecular weight pyrazines and a pyrrole. J. Agr. Food Chem., 14, 454-460.

[7] Miller (A.), Scanlan (R. A.), Lee (J. S.), Libbey (L. M.) and Morgan (M. E.) (1973). - Volatile compounds produced in sterile fish muscle by Pseudomonas perolens. Appl. Microbiol., 25, 257-261.

[8] Morgan (M. E.), Libbey (L. M.) and Scanlan (R. A.) (1972). - Identity of the musty potato aroma compound in milk cultures of Pseudomonas taetrolens. J. Dairy Sci., 55, 666.

[9] Murray (K. E.), Shipton (J.) and Whitfield (F. B.) (1970). - 2-methoxypyrazines and the flavour of green peas. Chem. Ind. (4 July 1970), 897-898.

[10] Pittet (A. O.) and Hruza (D. E.) (1974). - Comparative study of flavor properties of thiazole derivatives. J. Agr. Food Chem., 22, 264-269.

[11] Seifert (R. M.), Buttery (R. G.), Guadagni (D. G.), Black (D. R.) and Harris (J. G.) (1970). - Synthesis of some 2-methoxy-3-alkylpyrazines with strong bellpepper-like odors. J. Agr. Food Chem., 18, 246-249.

[12] Tressl (R.), Kossa (T.) and Renner (R.) (1974). - Uber die Bildung von Aromastoffen in Malz, Würze und Bier. Mschr. Brauerei, 27, 98-102.

[13] Van PraAg (M.), Stein (H. S.) and TibbetTS (M. S.) (1968). - Steam volatile aroma constituents of roasted cocoa beans. J. Agr. Food Chem., 16, 1005-1008.

[14] Dumont (J. P.), Roger (Sylviane) et AdDA (J.) (1974). - Etude des composés volatils neutres présents dans les fromages à pâte molle et à croûte lavée. Le Lait, 54, 31-43. 